\title{
ARTICLE Deficient inhibition in alcohol-dependence: let's consider the role of the motor system!
}

\author{
Caroline Quoilin ${ }^{1,2}$, Emmanuelle Wilhelm ${ }^{1}$, Pierre Maurage ${ }^{2,3}$, Philippe de Timary $^{1,2,4,5}$ and Julie Duque ${ }^{1,2}$
}

\begin{abstract}
Impaired inhibitory control contributes to the development, maintenance, and relapse of alcohol-dependence, but the neural correlates of this deficit are still unclear. Because inhibitory control has been labeled as an executive function, most studies have focused on prefrontal areas, overlooking the contribution of more "primary" structures, such as the motor system. Yet, appropriate neural inhibition of the motor output pathway has emerged as a central aspect of healthy behavior. Here, we tested the hypothesis that this motor inhibition is altered in alcohol-dependence. Neural inhibitory measures of motor activity were obtained in 20 detoxified alcohol-dependent (AD) patients and 20 matched healthy subjects, using a standard transcranial magnetic stimulation procedure whereby motor-evoked potentials (MEPs) are elicited in a choice reaction time task. Moreover, behavioral inhibition and trait impulsivity were evaluated in all participants. Finally, the relapse status of patients was assessed 1 year after the experiment. As expected, AD patients displayed poorer behavioral inhibition and higher trait impulsivity than controls. More importantly, the MEP data revealed a considerable shortage of neural motor inhibition in AD patients. Interestingly, this neural defect was strongest in the patients who ended up relapsing during the year following the experiment. Our data suggest a strong motor component in the neural correlates of altered inhibitory control in AD patients. They also highlight an intriguing relationship with relapse and the perspective of a new biomarker to follow strategies aiming at reducing relapse in AD patients.
\end{abstract}

Neuropsychopharmacology (2018) 43:1851-1858; https://doi.org/10.1038/s41386-018-0074-0

\section{INTRODUCTION}

Loss of control over drinking is a core element of alcoholdependence and is thought to partially result from an inability to inhibit prepotent but inappropriate responses [1]. Accordingly, several lines of evidence indicate that deficient inhibitory control contributes to the development and maintenance of the disorder, and augments the propensity of relapse, even after years of abstinence $[2,3]$.

The ability to inhibit prepotent responses, commonly called response inhibition, has been recognized as a critical executive function supporting goal-directed behaviors. Consequently, inhibitory control has been perceived as a pure cognitive entity, mainly relying on the structural and functional integrity of the prefrontal cortex [4]. However, recent works indicate that processes involving the motor system may also contribute to inhibitory control, with a large body of literature demonstrating that successful inhibition entails a decrease in motor system excitability [5-7]. Though, these mechanisms have been totally ignored in previous studies on alcohol-dependent (AD) patients.

When applied over the hand area of the primary motor cortex (M1), single-pulse transcranial magnetic stimulation (TMS) can elicit motor-evoked potentials (MEPs) in targeted finger muscles. Interestingly, the amplitude of MEPs is strongly reduced preceding voluntary movements relative to resting conditions, reflecting the operation of powerful inhibitory influences that suppress motor activity during action preparation [8-12]. One predominant view has been that this neural motor inhibition shapes brain activity to favor goal-directed behaviors over premature or inappropriate motor responses [5].

Because inhibition of the motor output system is thought to support the ability to subjugate inappropriate behaviors and because this aptitude is altered in alcohol-dependence, we tested the hypothesis that $A D$ patients suffer from a shortage of neural motor inhibition during action preparation. Moreover, we evaluated the potential relationship between the level of neural motor inhibition and the propensity to relapse within the following year. Finally, we also assessed trait impulsivity and behavioral inhibition, using questionnaires and neuropsychological tasks.

\section{MATERIALS AND METHODS}

Participants

Twenty AD patients ( 11 women; $51.1 \pm 6.93$ years old), diagnosed by a psychiatrist according to DSM-IV criteria, were tested during the third week of their detoxification program (St. Luc Academic Hospital, Université catholique de Louvain, Brussels, Belgium). They were abstinent for 17 to 20 days and no longer on withdrawal medication. Their mean alcohol consumption before detoxification was 19.9 alcohol units $(S D=14.33$ ) per day (an alcohol unit $=10 \mathrm{~g}$ of pure ethanol), and their mean duration of

\footnotetext{
${ }^{1}$ Institute of Neuroscience, Université Catholique de Louvain, Brussels, Belgium; ${ }^{2}$ Laboratory for Experimental Psychopathology, Psychological Sciences Research Institute, Université Catholique de Louvain, Louvain-la-Neuve, Belgium; ${ }^{3}$ Research Institute for Psychological Sciences, Université Catholique de Louvain, Louvain-la-Neuve, Belgium; ${ }^{4}$ Unité Intégrée d'Hépatologie, Saint-Luc Academic Hospital, Université Catholique de Louvain, Brussels, Belgium and ${ }^{5}$ Department of Adult Psychiatry, Saint-Luc Academic Hospital, Université Catholique de Louvain, Brussels, Belgium

Correspondence: Caroline Quoilin (caroline.quoilin@uclouvain.be)
}

Received: 12 December 2017 Revised: 30 March 2018 Accepted: 14 April 2018

Published online: 26 April 2018 


\section{A. Blank}
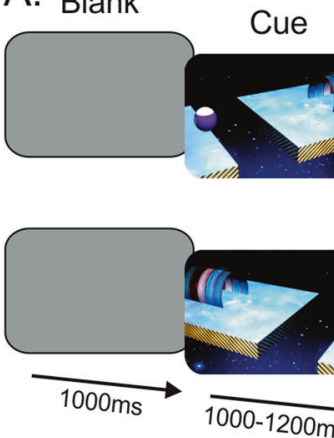
Cue Imperative

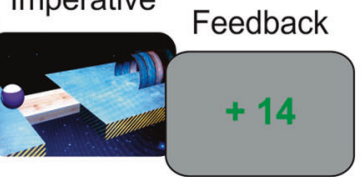

B.
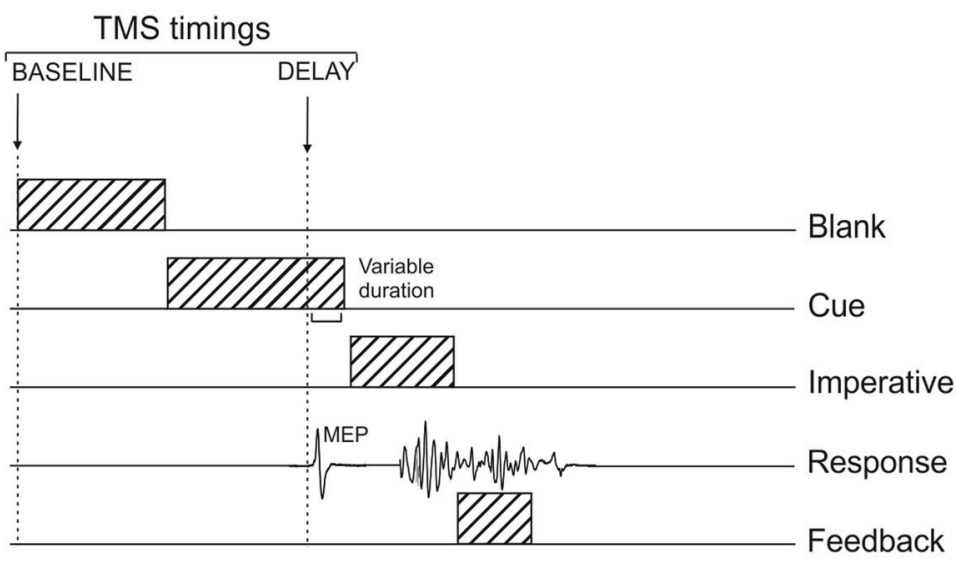

C.

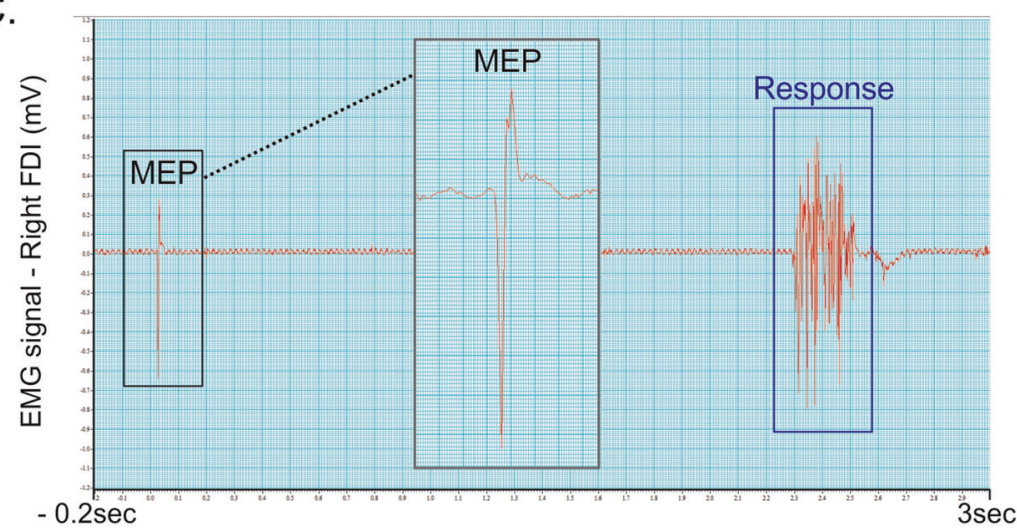

Fig. 1 Setup to measure neural motor inhibition. a Instructed-delay choice reaction time (RT) task. Subjects were required to perform an abduction movement of the left (upper trace) or right (lower trace) index finger in order to "shoot a ball into a goal." The required response was indicated by the position of the ball (preparatory cue) which appeared on the left or right side of the screen. Importantly, subjects had to withhold their response until the onset of a bridge (imperative signal). Then, they had to respond as fast as possible (RT of $700 \mathrm{~ms}$ max). Finally, a feedback score reflecting the performance was displayed for $500 \mathrm{~ms}$. The RT was defined as the time interval between the onset of the imperative signal and the moment when the index finger touched the inner edge of a response device specifically designed to control for the occurrence of premature responses (schematic representation of the device on the upper trace and actual photography on the lower trace). b TMS timings. One single TMS pulse was delivered in each trial over the left or the right M1 (separate blocks) at two possible timings:

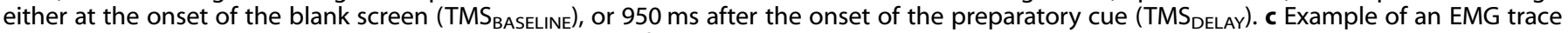
showing a MEP elicited at TMS collected for $3200 \mathrm{~ms}$ on each trial, starting $200 \mathrm{~ms}$ before the TMS pulse. The size of the MEP is enlarged in the inset for better visualization. FDI first dorsal interosseous, TMS transcranial magnetic stimulation, MEP motor-evoked potential, M1 primary motor cortex

alcohol-dependence was 14.6 years $(S D=8.93)$. On the basis of the Edinburgh Handedness Inventory [13], 18 were right-handed. ADs were matched for age, gender, and education level with 20 right-handed control subjects; their mean alcohol consumption was 0.4 units $(S D=0.71)$ per day. Exclusion criteria for both groups included major neurological or psychiatric disorder, any drug treatment that could influence performance or neural activity (including benzodiazepine), and no history of polysubstance abuse (except nicotine). Nicotine dependence was more prevalent among ADs than controls ( $n=12$ and $n=3$, respectively; $X^{2}=$ 8.64; $p<0.01$ ), as already shown in the past [14]. All participants gave written informed consent, following a protocol approved by the Biomedical Ethical Committee of the Saint-Luc University Hospital, Université catholique de Louvain. A financial compensation of $€ 50$ was only provided to the controls, in accordance with ethical regulations. 
Experimental procedure

Self-reported measures. Current clinical status was measured using French versions of the Spielberger State Trait Anxiety Inventory (STAI Trait and State; $[15,16])$ and the Beck Depression Inventory (BDI; $[17,18])$. Trait impulsivity was evaluated using the UPPS Impulsive Behavior scale [19, 20].

Behavioral measures. Task details are provided in the Supplementary Materials and Methods. Briefly, participants performed a simple visual reaction time (RT) task and three neuropsychological Miyake tasks, including the Stop-Signal, the Anti-Saccade and the Number-Letter tasks. According to the theory of Miyake et al. [21], the first two tasks specifically address response inhibition (ability to inhibit prepotent responses), whereas the Number-Letter task evaluates the capacity to shift between mental sets, i.e. an executive function distinct from inhibition. The critical measure for each task was (1) the "stop-signal inhibition errors", defined as the percentage of responses occurring in stop-signal trials, (2) the "anti-saccade cost", defined as the RT difference between the antisaccade and control blocks, and (3) the "shifting cost", corresponding to the RT difference between the blocks requiring a mental shift or not. All tasks were implemented using E-Prime 2.0. (Psychology Software Tools, Inc.) and run on a computer. Participants were always required to respond as fast as possible with their dominant hand.

Neural motor inhibition measures. Participants sat in front of a computer screen with forearms resting in a semi-flexed position and hands placed palms down on a homemade response device. They performed an instructed-delay choice RT task, requiring them to choose between responding with a left or right index finger abduction according to the position of a preparatory cue (i.e., left or right-side ball separated from a goal by a gap), and to provide their response as quickly as possible after the onset of an imperative signal (i.e. bridge connecting the platforms on which the ball and the goal stood). Once a correct response was detected, the ball rolled over the bridge to reach the goal. Importantly, responses provided before the imperative signal caused the ball to fall into the gap.

The sequence of events of a typical trial is shown on Fig. 1a. Each trial started with the presentation of a blank screen for 1000 $\mathrm{ms}$. Then, the preparatory cue was displayed, allowing participants to prepare their movement. After a random period of 1000-1200 $\mathrm{ms}$, the imperative signal appeared and remained visible until a finger response was detected (700 ms max). Finally, a feedback score reflecting the performance appeared for $500 \mathrm{~ms}$ : correct responses led to positive scores (inversely proportional to the RT, ceiling at +25 ) whereas errors resulted in a fixed negative score $(-15)$.

Each subject performed 4 blocks of 56 trials with one TMS pulse applied on each trial (at $115 \%$ of the resting motor threshold), over the left (mean intensity of $51.75 \%$ and $56.35 \%$ of the maximum stimulator output in controls and ADs, respectively) or the right $\mathrm{M} 1$ (49.45\% and 52.9\%, respectively). MEPs were elicited in the first dorsal interosseous (FDI) of the dominant (MEP $)$ or non-dominant $\left(\right.$ MEP $\left._{\mathrm{ND}}\right)$ hand (separate blocks). The TMS pulse could occur either at the onset of the blank screen, when subjects were at rest $\left(\mathrm{TMS}_{\text {BASELINE; }} 18 \mathrm{MEPs} /\right.$ block), or during the delay period, $950 \mathrm{~ms}$ after the onset of the preparatory cue (TMS DELAY; 38 MEPs/block), when subjects were withholding their response (Fig. 1b). At the latter timing, MEPs could either occur in a hand cued for the forthcoming response (selected condition; e.g., MEP $_{\mathrm{ND}}$ in ND hand trials; 19 MEPs/block) or in the non-cued hand (non-selected condition; e.g., MEP ${ }_{N D}$ in $\mathrm{D}$ hand trial; $19 \mathrm{MEPs} /$ block). Electromyography (EMG) was recorded from both FDIs (Fig. 1c). Details about the TMS and EMG procedures are provided in the Supplementary Materials and Methods.
Relapse measures. Twelve months after the experiment, ADs were called back and questioned about their drinking behavior since their detoxification program. Patients were classified as "relapsers" when they had consumed at least $60 \mathrm{~g}$ alcohol (male; $40 \mathrm{~g}$ for a female) on a single occasion during the past year, in accordance with criteria recommended in standard clinical trials [22-24]. The one-year time point was chosen as it has been shown to be a strong predictor of subsequent alcohol use $[25,26]$. Of the 20 patients, 11 had resumed consumption, while two women reported having consumed one or more sips of alcohol without reaching the threshold of $40 \mathrm{~g}$ alcohol and were therefore included in the non-relapser group $(n=9)$.

\section{Statistical analyses}

Demographic variables and current clinical status in ADs and controls were compared using independent t-tests, and those found to significantly differ between groups were included as covariates in the following analyses to control for their influence. Trait impulsivity was analyzed by conducting a multivariate analysis of variance (MANOVA) on scores reported at the four subscales of the UPPS questionnaire. Data from behavioral and neural motor inhibition were analyzed using analyses of covariance (ANCOVAs), and the relationship between these variables was evaluated using partial Pearson's correlations. ANCOVAs were also used to compare the inhibitory profile of controls, non-relapsers and relapsers. The Fisher's least significant difference (LSD) method was used to run post-hoc comparisons. Statistical significance was set at $p<0.05$. Further details on statistical analyses are provided in the Supplementary Materials and Methods.

\section{RESULTS}

\section{Self-report measures}

Demographic and current clinical status. As illustrated in Table S1, ADs and controls were fully matched for age and education level. In addition, they did not significantly differ for state anxiety $\left(t_{38}=1.02 ; p=0.32\right)$. By contrast, ADs reported higher levels of trait anxiety $\left(t_{38}=4.07 ; p<0.001\right)$ and depression $\left(t_{38}=3.98\right.$; $p<0.001)$. Therefore, these factors were included as covariates in subsequent analyses. Importantly, despite the correlation between these two variables $(r=0.77)$, collinearity was excluded given the magnitude of the variance inflation factor $(\mathrm{VIF}=2.37$, i.e., <10; [27]).

Trait impulsivity. The MANOVA performed on scores at the UPPS impulsivity scale showed a significant main effect of GROUP $\left(\lambda_{4,33}=0.73 ; p<0.05\right)$, due to higher scores on the urgency subscale in $\operatorname{ADs}\left(F_{1,36}=4.81 ; p<0.05\right.$; Table $\left.S 2\right)$.

\section{Behavioral measures}

RTs in ADs (mean $=313.5$ ) and controls (mean $=300.6$ ) were not significantly different in the simple visual RT task $\left(F_{1,36}=1.18\right.$; $p=0.28$ ). Regarding the neuropsychological tasks, the main finding was that ADs displayed a larger anti-saccade cost than controls $\left(F_{1,36}=8.32 ; p<0.01\right.$; Fig. $\left.2 b\right)$, indicating that it was more difficult for them to inhibit the initial reflexive saccade towards the incongruent visual cue (but see also our comment in the Supplementary Materials and Methods). Besides, the percentage of inhibition errors in the stop-signal task $\left(F_{1,35}=1.03 ; p=0.32\right.$; Fig. $2 \mathrm{a})$ and the shifting cost in the number-letter task $\left(F_{1,36}=0.08\right.$; $p=0.77$; Fig. $2 \mathrm{c}$ ) were comparable in ADs and controls. More details on analyses performed in each task are provided in the Supplementary Materials and Methods.

Neural motor inhibition measures

MEP data. Overall, MEPs elicited at $\mathrm{TMS}_{\text {BASELINE }}$ were smaller in ADs $(1.03 \pm 0.25 \mathrm{mV})$ than in controls $(1.84 \pm 0.23 \mathrm{mV})$, as indicated 
A. Stop Signal

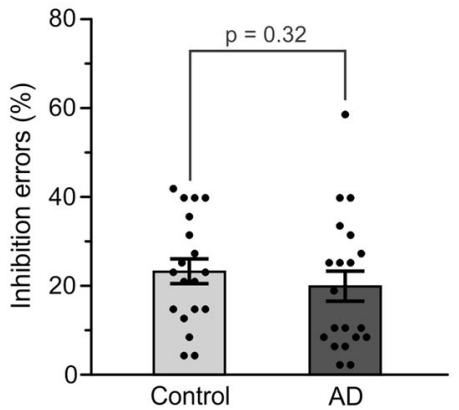

B. Anti-Saccade

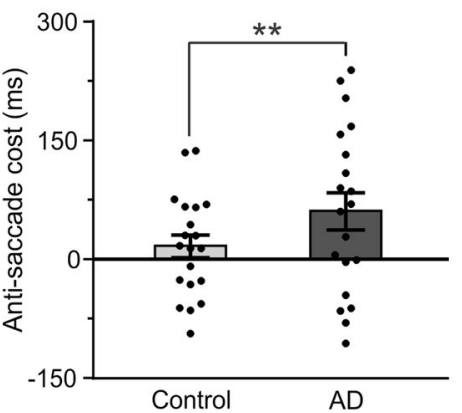

C. Number-Letter

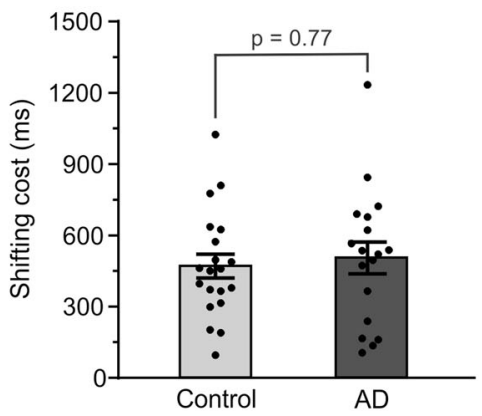

Fig. 2 Neuropsychological Miyake tasks. The unadjusted means (gray bars) and individual data (black dots) are shown for the critical measures of each task in the control and alcohol-dependent (AD) groups. a Inhibition errors in the stop-signal trials, defined as the percentage of categorization responses occurring in the stop trials. b Anti-saccade cost (ms) in the anti-saccade task, defined as the difference between the average RTs in the incongruent block and the average RTs in the two first blocks, with ${ }^{* *} p<0.01$. c Shifting cost (ms) in the number-letter task, defined as the difference between the average RTs for the trials requiring a mental shift in the third block and the average RTs in the two other blocks. (a, b) Provided a measure of inhibition, whereas c provided a control measure of shifting abilities. Further details relative to the experimental procedure of these tasks are provided in the Supplementary Materials and Methods. Our results highlight a deficit in behavioral inhibition when $A D$ patients have to suppress an oculomotor response

A. Control subjects

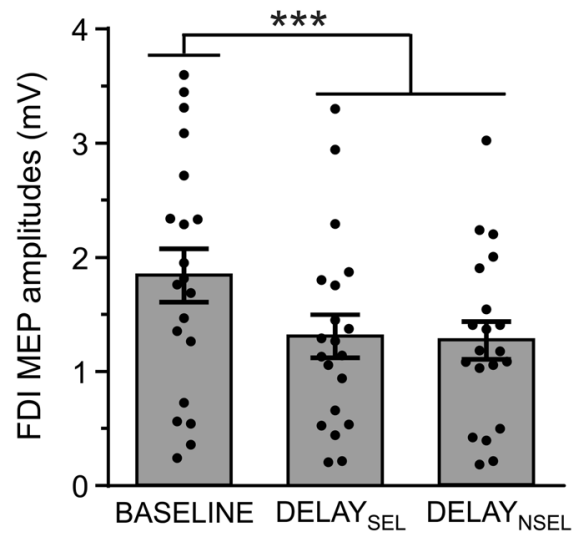

B. AD patients

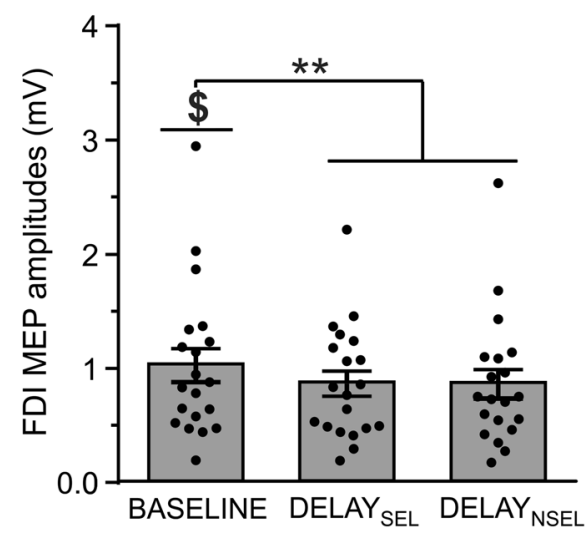

Fig. 3 Unadjusted means (gray bars) and individual data (black dots) are shown for the raw amplitude of motor-evoked potentials (MEPs) recorded in the first dorsal interosseous (FDI) in $\mathbf{a}$ control subjects and $\mathbf{b}$ alcohol-dependent $(\mathrm{AD})$ patients. MEPs were elicited during the intertrial interval (BASELINE) or during the delay period in a hand that was either selected (DELAY $\mathrm{SEL}_{\text {) }}$ or non-selected (DELAY NSEL ) for the forthcoming action. As the main effect of MEP-SIDE was not significant, data are collapsed across both hands. ${ }^{* *} p<0.01$ and ${ }^{* * *} p<0.001$. $\$=$ MEPs at TMS DELAY $_{\text {NSEL }}$ in both control subjects and AD patients

by a main effect of GROUP $\left(F_{1,36}=5.82 ; p<0.05\right.$, Fig. 3), regardless of the hand within which MEPs were elicited (GROUP $\times$ MEP-SIDE interaction: $F_{1,36}=1.81 ; p=0.19$ ). This effect occurred even though the same TMS intensity was used in both groups, suggesting a lower corticospinal excitability at rest in ADs.

As shown on Fig. 3a, in controls, MEPs were systematically smaller at TMS DELAY relative to TMS BASELINE (CONDITION: $\left.F_{2,38}=4.07 ; p<0.001\right)$, whether elicited in a selected or nonselected condition (both $p<0.001$ ), consistent with the occurrence of neural motor inhibition during action preparation. A suppression of MEPs at TMS DELAY was also found in $A D s$, as indicated by a main effect of CONDITION $\left(F_{2,38}=6.69 ; p<0.01\right.$; Fig. 3b). Similar to controls, this effect occurred regardless of whether MEPs were elicited in a selected or non-selected condition (both $p<0.01$ ).

In order to compare the strength of MEP suppression between both groups (Fig. 4), analyses were run on MEPs at TMS $S_{\text {DELAY }}$ expressed in percentage of MEPs elicited at TMS BASELINE (i.e.,
$\left.\left[\mathrm{TMS}_{\text {DELAY }} / \mathrm{TMS}_{\text {BASELINE}}\right] \times 100\right)$. Interestingly, MEP suppression was attenuated in ADs compared to controls (GROUP; $F_{1,36}=6.97 ; p<$ 0.05 ). This effect is unlikely to be due to the MEP difference at

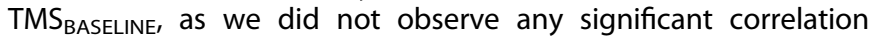
between normalized MEPs at TMS at TMS $_{\text {BASELINE }}$ (all $-0.37<r<-0.07$ and $p>0.11$ ). Intriguingly, the GROUP effect depended on the hand within which MEPs were elicited (GROUP $\times$ MEP-SIDE interaction; $F_{1,36}=4.06 ; p<0.05$ ); it was observed for the $\operatorname{MEP}_{\mathrm{ND}}(p<0.01$; Fig. 4a) but not for the $\operatorname{MEP}_{\mathrm{D}}(p=0.35$; Fig. $4 \mathrm{~b})$. Relatedly, the MEP suppression tended to be more pronounced in the non-dominant than in the dominant hand of controls $(p<0.09)$, whereas this difference was not observed in ADs $(p=0.23)$, with MEPs at TMS DELAY showing very little suppression in both hands of ADs. Finally, neither the factor CONDITION $\left(F_{1,36}=0.52 ; p=0.48\right)$ nor the GROUP $\times$ CONDITION interaction $\left(F_{1,36}=0.03 ; p=0.87\right)$ were significant, suggesting that

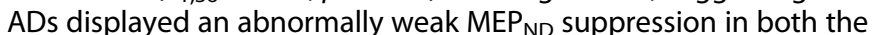
selected and non-selected conditions. 
A. MEPs in a non-dominant hand

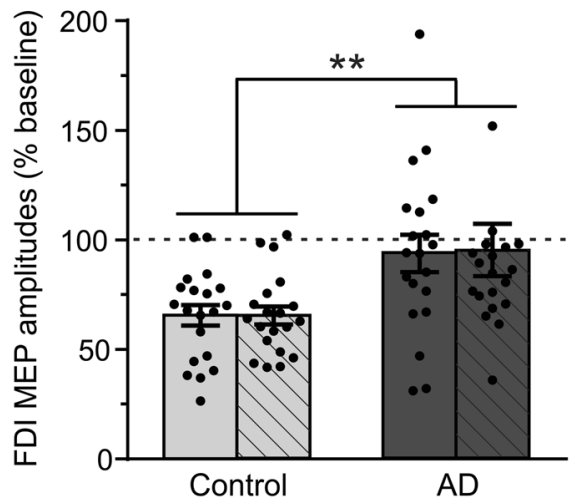

B. MEPs in a dominant hand

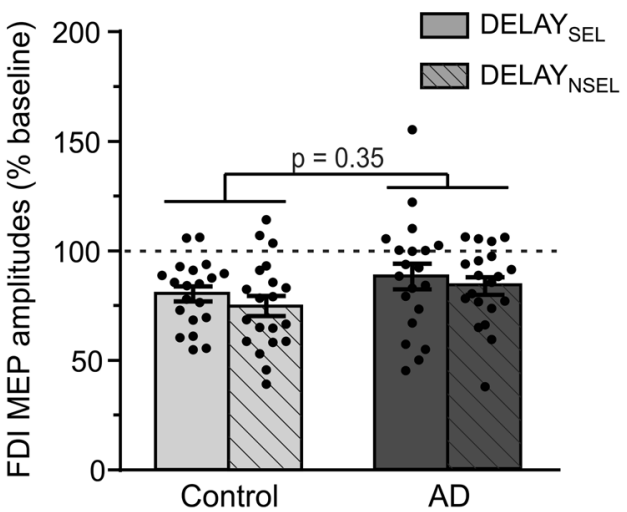

Fig. 4 Unadjusted means (gray bars) and individual data (black dots) are shown for the amplitude of motor-evoked potentials (MEPs)

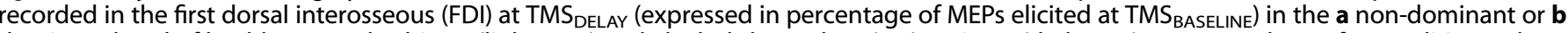
dominant hand of healthy control subjects (light gray) and alcohol-dependent (AD) patients (dark gray). MEPs are shown for conditions where the muscle from which they were recorded was either selected (DELAY SEL; $_{\text {; open bars) or non-selected (DELAY }}$ NSEL; dashed bars) for the forthcoming response. Please note that one AD patient was not displayed for the condition "DELAY ${ }_{\mathrm{SEL}}$ " in the non-dominant hand due to its extreme value $(X=301.5)$. This patient is nevertheless considered in the mean value. Importantly, exclusion of this participant from the analyses would have led to the same outcomes. ${ }^{* *} p<0.01$. Our results show an attenuated suppression of MEP ${ }_{N D}$ for both the DELAY ${ }_{S E L}$ and DELAY NSEL Conditions

Taken all together, those results indicate an alteration of neural motor inhibition in AD patients compared to healthy subjects, especially with MEPs elicited in the non-dominant hand.

Behavioral data. As illustrated in Table S3, ADs and controls performed similarly on the choice RT task; they displayed comparable RTs $\left(F_{1,36}=0.53 ; p=0.47\right)$ and an equivalent percentage of anticipation and hand choice errors $\left(F_{1,36}=0.89 ; p=0.35\right.$ and $F_{1,36}=0.10 ; p=0.76$; respectively).

Relationship between the different measures of inhibition The results reported above reveal a deficit in inhibitory control in $A D$ patients at several levels. First, the urgency subscale scores were higher than in controls, reflecting a larger trait impulsivity. Second, ADs displayed a greater anti-saccade cost, implying a lack of behavioral inhibition. Finally, we observed an abnormally weak

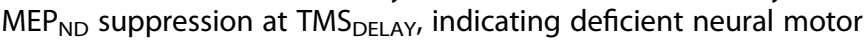
inhibition. Notably, we did not find any significant correlation between these three types of deficits in ADs (all $-0.18<r<0.22$ and $p>0.39$ ).

Relationship between deficient inhibition and relapse

The measures of trait impulsivity (urgency score), behavioral inhibition (anti-saccade cost) and neural motor inhibition (MEP suppression) were used to evaluate the potential connection between deficient inhibition and relapse. To do so, patients were separated according to whether they had relapsed $(n=11)$ or not $(n=9)$ and were compared to controls. As shown on Fig. 5, analyses revealed a significant main effect of GROUP on all three variables (trait impulsivity: $F_{2,37}=10.52 ; p<0.001$; behavioral inhibition: $F_{2,35}=8.99 ; \quad p<0.001$; neural motor inhibition: $\left.F_{2,35}=6.53 ; p<0.01\right)$. Interestingly, patients who ended up relapsing displayed poorer behavioral inhibition as well as weaker motor suppression during action preparation than those who remained sober $(p<0.01$ and $p<0.05$, respectively). In fact, the abstinent patients showed similar levels of behavioral and neural motor inhibition to controls ( $p=0.58$ and $p=0.28$, respectively), whereas those who relapsed were altered on both measures $(p<0.01$ and $p<0.001$, respectively). Such an effect was not observed for trait impulsivity: the latter was higher in patients than in controls, regardless of the relapse status (both $p<0.01)$.

\section{DISCUSSION}

The development, maintenance, and relapse of substance-use disorders have usually been related to a lack of inhibitory control. This deficit is largely documented in AD patients, with a rich body of literature reporting significant alterations commonly evidenced using self-reported questionnaires and neuropsychological tasks [1]. Moreover, neuroimaging studies have reported abnormalities in prefrontal networks that are generally associated with inhibitory control $[28,29]$. Here, we used TMS to address the question of inhibitory deficits from a different perspective, focusing on a potential dysfunction of motor circuits, given the recent evidence that these structures display strong inhibitory changes during action preparation and that these modulatory effects are thought to support healthy goal-directed behaviors [5]. Our data indicate that behavioral inhibitory difficulties of $A D$ patients occur in parallel with a strong lack of neural motor inhibition during action preparation. Critically, both behavioral and neural motor inhibition deficits seem to be related to the risk of relapse.

\section{Neural motor inhibition}

Many previous TMS studies have shown that the motor system is strongly suppressed during action preparation [9-11, 30]. Consistent with this literature, in controls, MEPs elicited at TMS $S_{\text {DELAY }}$ were substantially smaller than MEPs elicited at

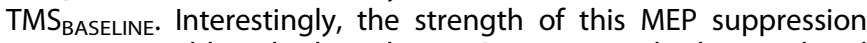
was comparable whether the MEPs were probed in a hand selected or non-selected for the subsequent action, which is line with recent works inferring the existence of a generic form of inhibition targeting all muscle representations regardless of their function in the forthcoming response [5]. MEPs were also reduced in $A D$ patients, although the strength of this change was relatively weak. Accordingly, the MEP suppression was significantly smaller in $A D$ s than in controls, suggesting that $A D$ patients suffer from an alteration in neural motor inhibition. This attenuated MEP suppression cannot be attributed to a lower involvement of ADs in the task, as they performed equally well as controls.

The shortage of neural motor inhibition in ADs was observed when MEPs were elicited in the non-dominant hand. Interestingly, in controls, the MEP suppression tended to be stronger in that hand relative to the dominant hand, which suggests that the motor pathway controlling the non-dominant limb displays more inhibitory influences than that controlling the dominant one, as 
A. Urgency (UPPS scale)

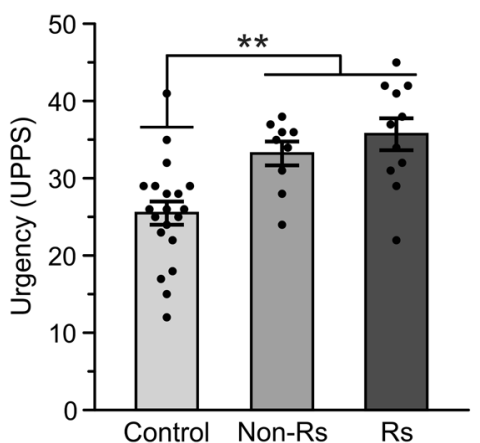

B. Anti-saccade cost

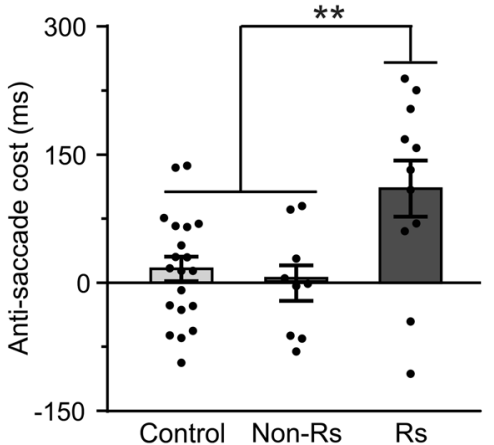

C. MEPs in a non-dominant hand

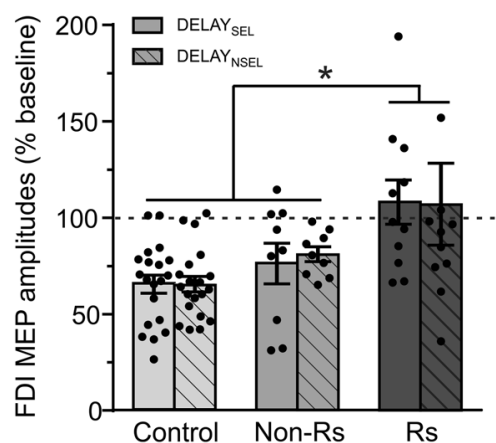

Fig. 5 Unadjusted means (gray bars) and individual data (black dots) are shown for a trait impulsivity (i.e., scores of the urgency subscale of the UPPS impulsive behavior scale), b behavioral inhibition (i.e., anti-saccade cost) and $\mathbf{c}$ neural motor inhibition (i.e., motor-evoked potentials

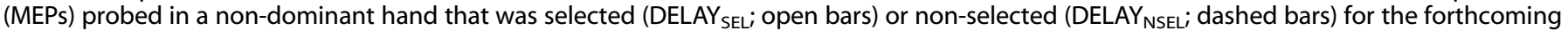
response) in control subjects and in alcohol-dependent patients who did not or did relapse (Non-Rs and Rs, respectively) during the year following the experiment. Please note that the anti-saccade cost and MEPs probed in the first dorsal interosseous (FDI) were corrected for the influence of covariates, and that one "Rs" patient was not displayed for the condition "DELAY SEL" due to its extreme value $(X=301.5)$. This patient is nevertheless considered in the mean value. Importantly, exclusion of this participant from the analyses would have led to the same outcomes. ${ }^{*} p<0.05$ and ${ }^{* *} p<0.01$

already discussed in the past $[12,31,32]$. Thus, deficient neural motor inhibition in ADs concerns the condition where MEP suppression is normally the strongest.

Notably, MEPs at TMS controls. The question arises as to whether this difference may be responsible for the attenuated MEP suppression in patients: baseline MEPs might have been too small in ADs to show a further suppression at $\mathrm{TMS}_{\text {DELAY }}$ (floor effect). Although we cannot completely rule out this possibility, we believe it is unlikely. First, we did not find any relationship between the amplitude of MEPs at TMS $_{\text {BASELINE }}$ and the strength of MEP suppression at

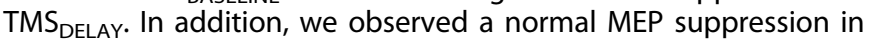
the dominant hand of ADs, although baseline MEPs were as small in that hand as in the non-dominant hand where the deficit was observed.

\section{Behavioral inhibition}

ADs displayed deficient behavioral inhibition, as evident from their lower performance in the anti-saccade task (but see also our comment in the Supplementary Materials and Methods). By contrast, they performed equally well as controls in the number-letter task, suggesting that inhibitory deficits occurred in the absence of major alterations in general executive functioning. Importantly, inhibitory deficits cannot be explained by a general psychomotor slowness sometimes reported in ADs [29, 33], as we did not observe any betweengroup difference in the simple RT task. Although prior neuropsychological works assessing response inhibition have led to equivocal findings [33-36], our data are in line with a recent meta-analysis validating the presence of behavioral inhibitory deficits in ADs [37].

One surprising result is the absence of difference between ADs and controls in the percentage of inhibition errors in the stopsignal task. However, this lack of effect could be due to our experimental procedure. As such, following the recommendations of Miyake and according to previous studies [21, 35, 38], the stopsignal delay was fixed; it corresponded to the mean RT in the first block minus $225 \mathrm{~ms}$. The fact that it was not constantly adjusted (as often the case) might have allowed participants lacking inhibition abilities to adopt a strategy whereby they maintained a high success rate by postponing response preparation. Consistently, patients displayed slower RTs than controls (see Supplementary Materials and Methods).
Trait impulsivity

ADs obtained higher scores than controls on the UPPS impulsive behavior scale, corroborating the well-documented link between impulsivity and alcohol-dependence [1]. The scores were particularly high on the urgency subscale, reflecting the tendency to act rashly under condition of negative affect. This form of impulsivity seems to be the one most strongly associated with psychopathologies [20], as again supported by our measures.

Relationship between the different measures of deficient inhibition

ADs were found to suffer from deficient inhibitory control at the neural, behavioral and trait levels. Interestingly, the strength of MEP suppression during action preparation did not correlate with urgency scores or with the anti-saccade cost, suggesting that neural motor inhibition reflects a different facet of inhibitory control. Besides, trait impulsivity and behavioral inhibition were not associated either, which is in line with previous meta-analytic works reporting a very slight overlap between these types of measure $[39,40]$.

\section{Deficient inhibition and relapse}

Interestingly, the $A D$ patients who relapsed within the year following the experiment were those displaying the strongest inhibitory deficits, both at the behavioral and neural level. In fact, the patients who remained sober responded as accurately as controls in the Anti-Saccade task, reflecting rather normal inhibitory skills. Moreover, the neural motor inhibition of abstinent patients seemed normal, as their MEP $_{\mathrm{ND}}$ suppression at $\mathrm{TMS}_{\text {DELAY }}$ was comparable to that displayed by healthy controls. Hence, deficient inhibition was only observed in subjects who ended up relapsing during the year following the experiment. Those results are in line with new evidence relating impaired inhibitory control and poor treatment outcomes in substance-use disorders [41]. In particular, by measuring event-related potentials [42] or behavioral performance $[43,44]$ in a Go/No-Go task in ADs, recent prospective studies have concluded that response inhibition abilities at treatment onset may predict future relapse.

By contrast, all patients displayed higher trait impulsivity compared to controls, regardless of the forthcoming relapse status. Interestingly, trait impulsivity represents a stable personality feature, whereas behavioral and neural motor inhibition are known to fluctuate over time and are therefore more prone to 
change $[10,45]$. For instance, deficient response inhibition has been found to improve following cognitive training [46], while MEP suppression can be strengthened with a training procedure of real-time neurofeedback [47]. As preliminary research also suggests that enhanced response inhibition might reduce subsequent alcohol intake [46], the development of training programs targeting behavioral and neural motor inhibition could therefore represent a promising approach in the treatment of alcohol-dependence.

\section{Conclusion and perspectives}

This study provides first evidence for the view that ADs suffer from a dysfunction of neural inhibitory mechanisms operating at the level of the motor output system. Interestingly, the strongest effects were reported in patients who ended up resuming consumption, suggesting that this deficit may contribute to the propensity to relapse. Our findings have important clinical implications. In particular, deficient neural motor inhibition could represent an objective TMS-based biomarker helping to detect people at high-risk of relapsing, as well as a promising target for pharmaceutical and training interventions.

New avenues of research are opened here. Notably, it would be interesting to include structural magnetic resonance imaging data to the TMS, neuropsychological and self-reported assessments in order to explore the neural bases of deficient motor inhibition. Moreover, the effect of alcohol, nicotine and comorbid dependence on response inhibition was not addressed in this study, and represents an appealing issue for future research. Finally, as alcohol-dependence is thought to result from impaired cognitive control associated with increased reactivity to alcohol-related stimuli, a next step will be to investigate the impact of these cues on the strength of neural motor inhibition.

\section{ACKNOWLEDGEMENTS}

We would like to thank Frédérique Casier for her involvement in the data collection and analyses. This work was supported by an incentive grant from the Belgian National Funds for Scientific Research (FRS-FNRS: MIS F.4512.14), the "Fonds Spéciaux de Recherche" (FSR) of the Université catholique de Louvain and the "Fondation Médicale Reine Elisabeth" (F.M.R.E.). CQ was funded by the FRS-FNRS and the "Fond de Recherche Clinique" of the Université catholique de Louvain. EW was funded by the FSR of the Universite catholique de Louvain. PM is a Senior Research Associate at the FRS-FNRS. PdT is a Senior Research Associate of the "Fond de Recherche Clinique" of the Universite catholique de Louvain. JD is a Senior Research Associate at the Institute of Neuroscience of the Université catholique de Louvain.

\section{ADDITIONAL INFORMATION}

The online version of this article (https://doi.org/10.1038/s41386-018-0074-0) contains supplementary material, which is available to authorized users.

Competing interests: The authors declare no competing interests.

Publisher's note: Springer Nature remains neutral with regard to jurisdictional claims in published maps and institutional affiliations.

\section{REFERENCES}

1. Wilcox CE, Dekonenko CJ, Mayer AR, Bogenschutz MP, Turner JA. Cognitive control in alcohol use disorder: deficits and clinical relevance. Rev Neurosci. 2014;25:1-24.

2. Domínguez-Salas S, Díaz-Batanero C, Lozano-Rojas OM, Verdejo-García A. Impact of general cognition and executive function deficits on addiction treatment outcomes: systematic review and discussion of neurocognitive pathways. Neurosci Biobehav Rev. 2016;71:772-801.

3. Verdejo-García A, Lawrence AJ, Clark L. Impulsivity as a vulnerability marker for substance-use disorders: review of findings from high-risk research, problem gamblers and genetic association studies. Neurosci Biobehav Rev. 2008;32:777-810.

4. Bari A, Robbins TW. Inhibition and impulsivity: behavioral and neural basis of response control. Prog Neurobiol. 2013;108:44-79.
5. Duque J, Greenhouse I, Labruna L, Ivry RB. Physiological markers of motor inhibition during human behavior. Trends Neurosci. 2017;40:219-36.

6. Bestmann S, Duque J. Transcranial magnetic stimulation: decomposing the processes underlying action preparation. Neuroscientist. 2016;22: 392-405.

7. Quoilin C, Derosiere G. Global and specific motor inhibitory mechanisms during action preparation. J Neurosci. 2015;35:16297-9.

8. Duque J, Labruna L, Cazares C, Ivry RB. Dissociating the influence of response selection and task anticipation on corticospinal suppression during response preparation. Neuropsychologia. 2014;65:287-96.

9. Duque J, Lew D, Mazzocchio R, Olivier E, Ivry RB. Evidence for two concurrent inhibitory mechanisms during response preparation. J Neurosci. 2010;30: 3793-802.

10. Greenhouse I, Saks D, Hoang T, Ivry RB. Inhibition during response preparation is sensitive to response complexity. J Neurophysiol. 2015;113:2792-800.

11. Quoilin C, Lambert J, Jacob B, Klein PA, Duque J. Comparison of motor inhibition in variants of the instructed-delay choice reaction time task. PLoS ONE. 2016;11: e0161964.

12. Wilhelm E, Quoilin C, Petitjean C, Duque J. A double-coil tms method to assess corticospinal excitability changes at a near-simultaneous time in the two hands during movement preparation. Front Hum Neurosci. 2016;10:88.

13. Oldfield RC. The assessment and analysis of handedness: the Edinburgh inventory. Neuropsychologia. 1971;9:97-113.

14. Grant BF, Hasin DS, Chou SP, Stinson FS, Dawson DA. Nicotine dependence and psychiatric disorders in the United States: results from the National Epidemiologic Survey on Alcohol and Related Conditions. Arch Gen Psychiatry. 2004;61:1107-15.

15. Bruchon-Schweitzer M, Paulhan I. Adaptation francophone de I'Inventaire d'Anxiété Trait-Etat (Forme Y) de Spielberger. 1st ed. Paris: Editions du Centre de Psychologie Appliquée; 1993.

16. Spielberger DC, Gorsuch RL, Lushene, Vagg PR, Jacobs GA. Manual for the Statetrait Anxiety Inventory. 1st ed. Palo Alto: Consulting Psychology Press; 1983.

17. Beck AT. A systematic investigation of depression. Compr Psychiatry. 1961;2:163-70.

18. Collet LL, Cottraux JJ. Inventaire abrégé de la dépression de Beck (13 items): Etude de la validité concurrente avec les échelles de Hamilton et de ralentissement de Widlöcher. Encéphale. 1986;12:77-9.

19. Billieux J, Rochat L, Ceschi G, Carré A, Offerlin-Meyer I, Defeldre AC, et al. Validation of a short French version of the UPPS-P Impulsive Behavior Scale. Compr Psychiatry. 2012;53:609-15.

20. Whiteside SP, Lynam DR, Miller JD, Reynolds SK. Validation of the UPPS Impulsive Behaviour Scale: a four-factor model of impulsivity. Eur J Pers. 2005;19:559-74.

21. Miyake A, Friedman NP, Emerson MJ, Witzki AH, Howerter A, Wager TD. The unity and diversity of executive functions and their contributions to complex "Frontal Lobe" tasks: a latent variable analysis. Cogn Psychol. 2000;41:49-100.

22. Sass H, Soyka M, Mann K, Zieglgänsberger W. Relapse prevention by acamprosate. Results from a placebo-controlled study on alcohol dependence. Arch Gen Psychiatry. 1996;53:673-80.

23. Seo S, Mohr J, Beck A, Wüstenberg T, Heinz A, Obermayer K. Predicting the future relapse of alcohol-dependent patients from structural and functional brain images. Addict Biol. 2015;20:1042-55.

24. Charlet K, Beck A, Jorde A, Wimmer L, Vollstädt-Klein S, Gallinat J, et al. Increased neural activity during high working memory load predicts low relapse risk in alcohol dependence. Addict Biol. 2014;19:402-14.

25. Maisto SA, Clifford PR, Stout RL, Davis CM. Drinking in the year after treatment as a predictor of three-year drinking outcomes. J Stud Alcohol. 2006;67: 823-32.

26. Maisto SA, Clifford PR, Stout RL, Davis CM. Moderate drinking in the first year after treatment as a predictor of three-year outcomes. J Stud Alcohol Drugs. 2007;68:419-27.

27. Hair JF, Anderson RE, Tatham RL, Black WC. Multivariate data analysis. 3rd edn New York: Macmillan; 1995

28. Li CS, Luo X, Yan P, Bergquist K, Sinha R. Altered impulse control in alcohol dependence: neural measures of stop signal performance. Alcohol Clin Exp Res. 2009;33:740-50.

29. Pandey AK, Kamarajan C, Manz N, Chorlian DB, Stimus A, Porjesz B. Delta, theta, and alpha event-related oscillations in alcoholics during Go/NoGo task: Neurocognitive deficits in execution, inhibition, and attention processing. Prog Neuropsychopharmacol Biol Psychiatry. 2016;65:158-71.

30. Duque J, Labruna L, Verset S, Olivier E, Ivry RB. Dissociating the role of prefrontal and premotor cortices in controlling inhibitory mechanisms during motor preparation. J Neurosci. 2012;32:806-16.

31. Duque J, Murase N, Celnik P, Hummel F, Harris-Love M, Mazzocchio R, et al. Intermanual differences in movement-related interhemispheric inhibition. J Cogn Neurosci. 2007;19:204-13. 
32. Leocani L, Cohen LG, Wassermann EM, Ikoma K, Hallett M. Human corticospinal excitability evaluated with transcranial magnetic stimulation during different reaction time paradigms. Brain. 2000;123:1161-73.

33. Lawrence AJ, Luty J, Bogdan NA, Sahakian BJ, Clark L. Impulsivity and response inhibition in alcohol dependence and problem gambling. Psychopharmacology. 2009;207:163-72.

34. Goudriaan AE, Oosterlaan J, de Beurs E, van den Brink W. Neurocognitive functions in pathological gambling: a comparison with alcohol dependence, Tourette syndrome and normal controls. Addiction. 2006;101: 534-47.

35. Maurage $P$, Callot $C$, Chang B, Philippot $P$, Rombaux $P$, de Timary P. Olfactory impairment is correlated with confabulation in alcoholism: towards a multimodal testing of orbitofrontal cortex. PLoS ONE. 2011;6:e23190.

36. Noël X, Van der Linden $M$, Brevers D, Campanella S, Verbanck P, Hanak C, et al. Separating intentional inhibition of prepotent responses and resistance to proactive interference in alcohol-dependent individuals. Drug Alcohol Depend. 2013;128:200-5.

37. Smith JL, Mattick RP, Jamadar SD, Iredale JM. Deficits in behavioural inhibition in substance abuse and addiction: a meta-analysis. Drug Alcohol Depend. 2014; 145:1-33.

38. Brion $M, D^{\prime}$ Hondt $F$, Pitel $A L$, Lecomte $B$, Ferauge $M$, de Timary $P$, et al. Executive functions in alcohol-dependence: a theoretically grounded and integrative exploration. Drug Alcohol Depend. 2017;177:39-47.

39. Lijfijt M, Bekker EM, Quik EH, Bakker J, Kenemans JL, Verbaten MN. Differences between low and high trait impulsivity are not associated with differences in inhibitory motor control. J Atten Disord. 2004;8:25-32.
40. Cyders MA, Coskunpinar A. Measurement of constructs using self-report and behavioral lab tasks: is there overlap in nomothetic span and construct representation for impulsivity? Clin Psychol Rev. 2011;31:965-82.

41. Stevens L, Verdejo-García A, Goudriaan AE, Roeyers $H$, Dom G, Vanderplasschen W. Impulsivity as a vulnerability factor for poor addiction treatment outcomes: a review of neurocognitive findings among individuals with substance use disorders. J Subst Abus Treat. 2014;47:58-72.

42. Petit G, Cimochowska A, Kornreich C, Hanak C, Verbanck P, Campanella S. Neurophysiological correlates of response inhibition predict relapse in detoxified alcoholic patients: some preliminary evidence from event-related potentials. Neuropsychiatr Dis Treat. 2014;10:1025-37.

43. Czapla M, Simon JJ, Richter B, Kluge M, Friederich HC, Herpertz S, et al. The impact of cognitive impairment and impulsivity on relapse of alcohol-dependent patients: implications for psychotherapeutic treatment. Addict Biol. 2016;21: 873-84.

44. Rupp Cl, Beck JK, Heinz A, Kemmler G, Manz S, Tempel K, et al. Impulsivity and alcohol dependence treatment completion: is there a neurocognitive risk factor at treatment entry? Alcohol Clin Exp Res. 2016;40:152-60.

45. Jones A, Christiansen P, Nederkoorn C, Houben K, Field M. Fluctuating disinhibition: implications for the understanding and treatment of alcohol and other substance use disorders. Front Psychiatry. 2013;4:140.

46. Houben K, Nederkoorn C, Wiers RW, Jansen A. Resisting temptation: decreasing alcohol-related affect and drinking behavior by training response inhibition. Drug Alcohol Depend. 2011;116:132-6.

47. Majid DS, Lewis C, Aron AR. Training voluntary motor suppression with real-time feedback of motor evoked potentials. J Neurophysiol. 2015;113:3446-52. 